\title{
Self-consistent stability analysis of spherical shocks
}

\author{
J. Sanz $\cdot$ S. Bouquet $\cdot$ M. Murakami
}

\begin{abstract}
In this paper, we study self-similar solutions, and their linear stability as well, describing the flow within a spherical shell with finite thickness, expanding according to a power law of time, $t^{q}$, where $q>0$. The shell propagates in a medium with initially uniform density and it is bounded by a strong shock wave at its outer border while the inner face is submitted to a time-dependent uniform pressure. For $q=2 / 5$, the well-known Sedov-Taylor solution is recovered. In addition, although both accelerated and decelerated shells can be unstable against dynamic perturbations, they exhibit highly different behaviors. Finally, the dispersion relation derived earlier by Vishniac (Vishniac, E.T. in Astrophys. J. 274:152, 1983) for an infinitely thin shell is obtained in the limit of an isothermal shock wave.
\end{abstract}

Keywords Supernova-remnants $\cdot$ Spherical shocks · Dense shell $\cdot$ Stability analysis

\section{Introduction}

The evolution and structure of supernova remnants (SNR's) result from the interaction between the ambient gas (circumstellar medium-CSM-and inter-stellar medium-ISM)

\section{J. Sanz ( $\square)$}

ETSI Aeronauticos, Universidad Politecnica de Madrid, Madrid 28040 , Spain

e-mail: franciscojavier.sanz@upm.es

\section{S. Bouquet}

CEA-DAM-DIF, 91297 Arpajon, France

M. Murakami

Institute of Laser Engineering, Osaka University, Osaka 565-0871, Japan surrounding the star that exploded and the plasma ejected (ejecta) by the star. According to their age and the type of supernova (SN), SNR's display a wide variety of size, morphology and appearance. Figure 1 shows the SNR Cassiopeia A. The star exploded about 300 years ago and the diameter of this remnant is about 10 light years. The bubble of emitting ejecta is enclosed within a almost spherical shock and, in addition, bright filaments, spherical caps or pieces of dense shells are clearly detected at the outer edge of the SNR.

Although in the intermediate time evolution of SNR's, the radius, $R(t)$, of the remnant is usually considered to evolve according to the Sedov solution, $R(t) \propto t^{2 / 5}$, (Se-

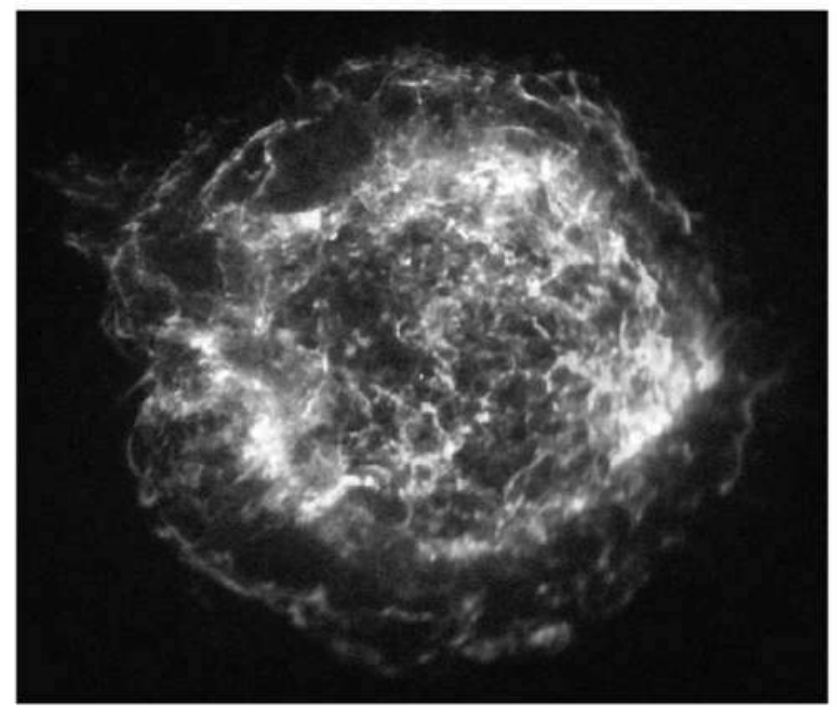

Fig. 1 The remnant from a supernova in Cassiopeia, which exploded $\sim 300$ years ago. The distance to this SNR is $\sim 3.4 \mathrm{kpc}$, and the diameter of this remnant is $\sim 10$ light year $(\sim 3 \mathrm{pc})$. Image from Chandra telescope (http://rst.gsfc.nasa.gov, section 20, Novae and Supernovae) 
dov 1959; Woltjer 1972; Gull 1973) the stability of the dense shell that encircle the ejecta is still an open question. The Rayleigh-Taylor instability (RTI) occurring in SRN's seems effective (Chevalier 1977, 1982; Chevalier et al. 1992; Chevalier and Blondin 1995) and it has been likely observed in Tycho SNR more than ten years ago (Velazquez et al. 1998). Besides, Vishniac and Ryu (Vishniac 1983, 1994; Ryu and Vishniac 1987, 1988, 1991; Vishniac and Ryu 1989) have shown theoretically that a rippled propagating decelerating shock front may experience pressure driven instabilities that produce a growth of the amplitude of the ripples. These predictions have been almost recovered numerically for specific classes of physical parameters/phenomena and for particular initial/boundary conditions (Mac Low and Norman 1993; Klein and Woods 1998; Cavet et al. 2009, 2010), however this process seems not yet clearly evidenced from astronomical observations.

In order to extend Sedov solution with additional analytical self-similar solutions (SSS's), we consider the expansion of a dense, compressible spherical shell of finite thickness in a uniform external medium. The shell is driven by a spherical piston which is assumed to correspond to the inner border of the shell moving according to $R_{\text {Pist }}(t) \propto$ $t^{q}$ where $q$ is an arbitrary positive exponent and where $R_{P i s t}(t)$ and $t$ stand respectively for the radius of the inner boundary of the shell and for the time. As the shell is over-dense compared to the outer medium (CSM and ISM) and to the inner flow, the shell experiences a density jump at each of its borders (inner and outer). Both the flows within the shell and in the medium enclosed by the shell are studied as well as the kind of instabilities experienced by the shell. RTI is likely expected to occur and we examine if the shell is stable against the amplification process as described by Vishniac and Ryu. In Sect. 2, the spherical model is presented and in Sect. 3 the one-dimensional time-dependent background flow is derived. Section 4 deals with the linear stability analysis in three dimensions and the various results are analyzed. Our conclusion is given in Sect. 5 .

\section{Spherical model}

We consider the dynamics of a spherical dense shell expanding in ISM. The density profile, $\rho_{a}(r)$, in the ambient gas (CSM plus ISM) surrounding the star is usually given by $\rho_{a}(r)=\rho_{0} r^{-s}$ (Chevalier 1982; Nadyozhin 1985; Chevalier et al. 1992) where $\rho_{0}$ is a constant and $s$ is an exponent satisfying $s \geq 0$. For $s=0$, the density is uniform and $\rho_{0}$ corresponds to the mass density of the ISM. The value $s=2$ is usually taken to describe the CSM, however in this paper we take into account the ISM only for

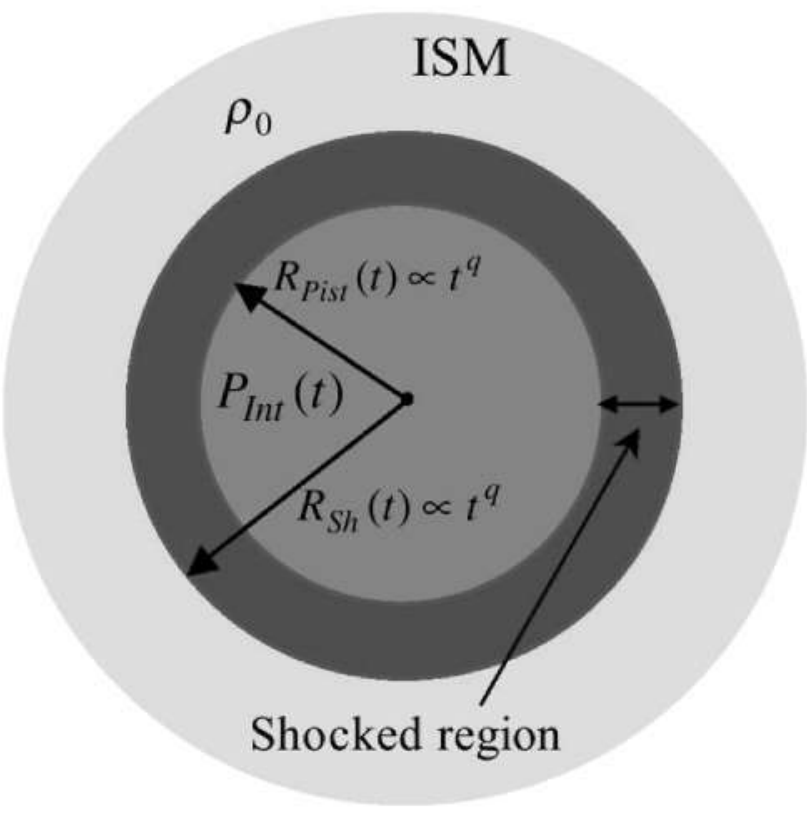

Fig. 2 Cartoon of the spherical dense shell model

the sake of simplicity. This is shown in Fig. 2. The formation of a thin dense shell in SNR's has been found to occur in the interaction zone between the SN ejecta and the surrounding medium. In addition, the interaction region is bounded by an outer shock wave (forward shock-FS) and by an inner shock wave (reverse shock-RS) as explained by Chevalier and coworkers (Chevalier et al. 1992; Chevalier and Blondin 1995; Wang and Chevalier 2001). Analytical solutions have been derived in this zone (Chevalier 1982) and the stability analysis of the shell in the infinitely thin thickness has been performed (Chevalier and Blondin 1995) for more general conditions than in the earlier work by Vishniac (1983).

In this paper, we study the dynamics of the dense shell with the help of SSS's assuming a spherical point explosion and we examine the stability of these solutions by accounting for the finite thickness of the shell. The time variation of the outer radius, $R_{S h}(t)$, of the shell is the same as for $R_{P i s t}(t)$, i.e.:

$R_{S h}(t) \propto R_{P i s t}(t) \propto t^{q}$.

When the exponent $q$ satisfies $q<1$, but $q>2 / 5$ - see below-(resp. $q>1$ ), the shell is decelerated (resp. accelerated). The material in the bubble is considered to be a hot, low-density gas with finite pressure $P_{\text {Int }}(t)$ while the external medium has a constant mass density (see above) with a zero temperature implying a zero thermal pressure in the ISM. Although this model is very simple, it clarifies several points especially about the stability analysis. 


\section{One dimensional background flow}

In one-dimensional spherical geometry, the equations governing the flow of an ideal gas (with polytropic index $\gamma$ ) within the shell are: mass, momentum and entropy conservation. Let $v(r, t), \rho(r, t), p(r, t)$ and $T(r, t)$ be the velocity, mass densily, pressure and temperature, respectively. The pressure and temperature obey the equation of state (EOS), $p(r, t)=\rho(r, t) k_{B} T(r, t) / m$, where $k_{B}$ is the Boltzmann constant and $m$ is the average mass per particle. At the outer border of the shell, $r=R_{S h}(t)$, the flow satisfies Rankine-Hugoniot equations in the strong shock approximation, i.c., $v=2 R_{S h}^{\prime}(t) /(\gamma+1), \rho=\rho_{0}(\gamma+1) /(\gamma-1)$, and $k_{B} T=2 m(\gamma-1)\left[R_{S h}^{\prime}(t) /(\gamma+1)\right]^{2}$, where the prime denotes the time derivative. On the other hand, the inner interface of the shell $\left(r=R_{P i s t}(t)\right)$ does not experience any mass flow and the pressure is continuous and hence, $v=R_{p_{i s t}}^{\prime}(t)$ and $p=P_{I n t}(t)$. Now, as we are looking for SSS's, we introduce dimensionless quantitics. The new "spatial" coordinate, is given by $\xi=r / R p_{i s t}(t)$, and its range of variation is $1 \leq \xi \leq \xi_{S h}$, where $\xi_{S h}=$ $R_{S h}(t) / R_{\text {Pist }}(t)$ is a constant. The self-similar dimensionless velocity, density, temperature and pressure are defined as $\bar{v}(\xi)=v(r, t) / R_{P_{i s t}}^{\prime}(t), \bar{\rho}(\xi)=\rho(r, t) / \rho_{0}, \bar{T}(\xi)=$ $k_{B} T(r, t) /\left[m R_{S h}^{\prime}(t)^{2}\right]$ and $\bar{p}(\xi)=\bar{\rho}(\xi) \bar{T}(\xi)$. Notice that the internal pressure $P_{I n t}(t)$ satisfies $P_{I n t}(t) \equiv$ $\rho_{0}\left[R_{\text {Pist }}^{\prime}(t)\right]^{2} \bar{p}(\xi=1) \propto t^{2(q-1)}$. This new set of variables transforms the system of partial differential equationsPDE's - within the shell in a system of ordinary differential equations-ODE's-that reads:

$$
\begin{aligned}
& {[\bar{v}(\xi)-\xi] \frac{d}{d \xi} \bar{\rho}(\xi)+\bar{\rho}(\xi) \frac{d}{d \xi} \bar{v}(\xi)+\frac{2}{\xi} \bar{\rho}(\xi) \bar{v}(\xi)=0,} \\
& {[\bar{v}(\xi)-\xi] \frac{d}{d \xi} \bar{v}(\xi)+\left(\frac{q-1}{q}\right) \bar{v}(\xi)} \\
& \quad=-\frac{1}{\bar{\rho}(\xi)} \frac{d}{d \xi}[\bar{\rho}(\xi) \bar{T}(\xi)], \\
& {[\bar{v}(\xi)-\xi] \frac{d}{d \xi}\left(\frac{\bar{T}(\xi)}{[\bar{\rho}(\xi)]^{\gamma-1}}\right)+2\left(\frac{q-1}{q}\right)\left(\frac{\bar{T}(\xi)}{[\bar{\rho}(\xi)]^{\gamma-1}}\right)} \\
& \quad=0 .
\end{aligned}
$$

The system of equations (2)-(4) is solved numerically for arbitrary values of $q$ and $\gamma$ with the boundary conditions:

$$
\begin{aligned}
& \bar{\rho}\left(\xi_{S h}\right)=(\gamma+1) /(\gamma-1), \\
& \bar{v}\left(\xi_{S h}\right)=[2 /(\gamma+1)] \xi_{S h}, \\
& \bar{T}\left(\xi_{S h}\right)=\left[2(\gamma-1) /(\gamma+1)^{2}\right]\left(\xi_{S h}\right)^{2},
\end{aligned}
$$

and

$$
\bar{v}(\xi=1)=1 \text {. }
$$

The solution of system (2)-(8) provides the strueture of the flow within the shell and the self-similar radius of the shock, $\xi_{S h}$. The dimensionless thickness of the shell does not depend on time and is given by:

$h=\frac{R_{S h}(t)-R_{P i s t}(t)}{R_{P i s t}(t)}=\xi_{S h}-1$.

From (2)-(4), it can be shown that the solution has a singular behavior close to the inner face of the shell $(\xi \rightarrow 1)$ given by $\bar{\rho}(1) \propto(\xi-1)^{-\mu}, \bar{T}(1) \propto(\xi-1)^{\mu}$ and $\bar{v}(1)-1 \propto \xi-1$, where the exponent $\mu(\mu=2(q-1) /[(2+3 \gamma) q-2])$ diverges for the critical value $q_{c}=2 /(2+3 \gamma)$, and for $\gamma=1$, we have $q_{c}=2 / 5$ while the upper limit of $\mu$ is $\mu_{\max }=2(q-1) /(5 q-2)$. Notice that the pressure is always finite at the inner surface of the shell but for $q>1$ (accelerated shock), the density goes to infinity and the temperature goes to zero whereas for a decelerated shock, $q_{c}<q<1$ (no solution exists for $q<q_{c}$-see below-and the upper value of $q_{c}$ corresponds to the Sedov-Taylor exponent, $q_{S T}=2 / 5$, since $\gamma$ satisfies $\gamma \geq 1$ ), the density goes to zero and the temperature goes to infinity. Actually, in the first situation, the density is monotonically decreasing between $R_{P i s t}(t)$ and $R_{S h}(t)$-sec Fig. 3-while it increases in the second case (Fig. 4).

Moreover, Fig. 5 shows that for a given value of $\gamma$, the thickness of the shell, $h(\gamma, q)$, increases as $q$ decreases. As indicated above, for $q \rightarrow 2 / 5, R_{\text {Pist }}(t) \rightarrow 0$ and no solution exists for $q<2 / 5$. In this configuration the inner bubble has a zero extension and the shell corresponds to a sphere with radius $R_{S h}(t)$. This flow is described by the well-known Scdov-Taylor solution (Sedov 1959) and it is exhibited in Fig. 6.

For arbitrary exponent $q$ the shell experiences a point explosion but the energy is not delivered instantaneously as the work achicved by the pressure forec is $E(t)=$

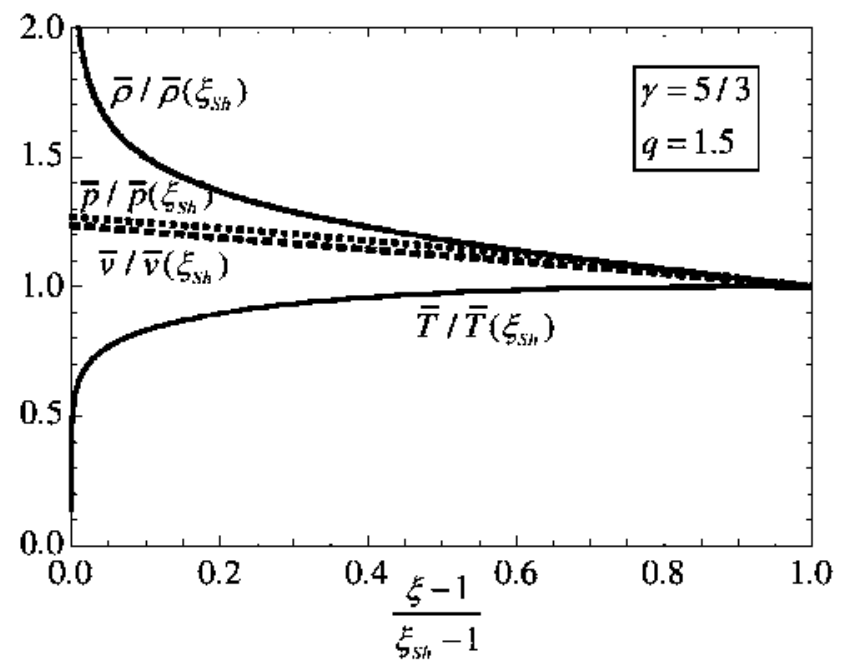

Fig. 3 Flow variables in the accelerated case $q=1.5$ 


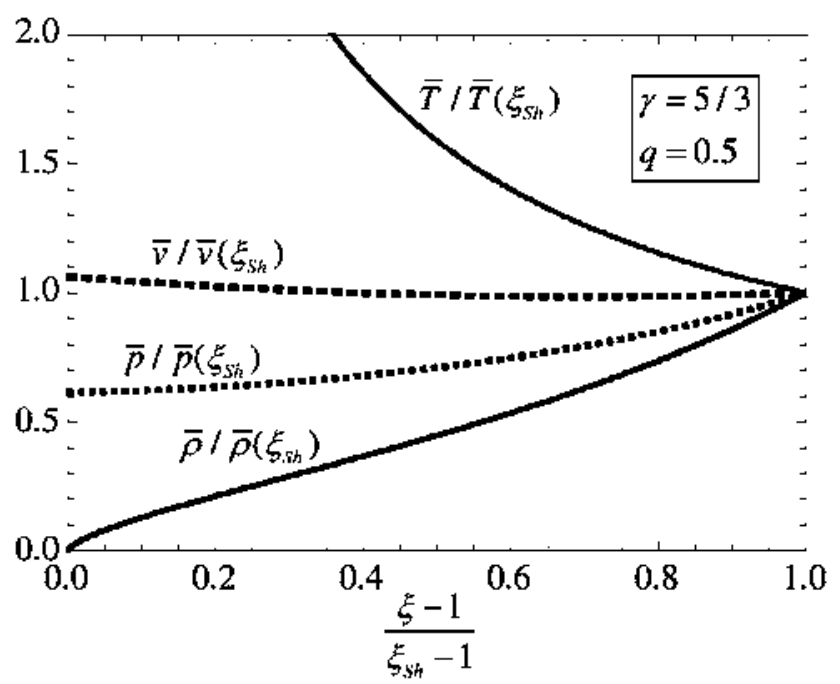

Fig. 4 Flow variables in the decelerated case $q=0.5$

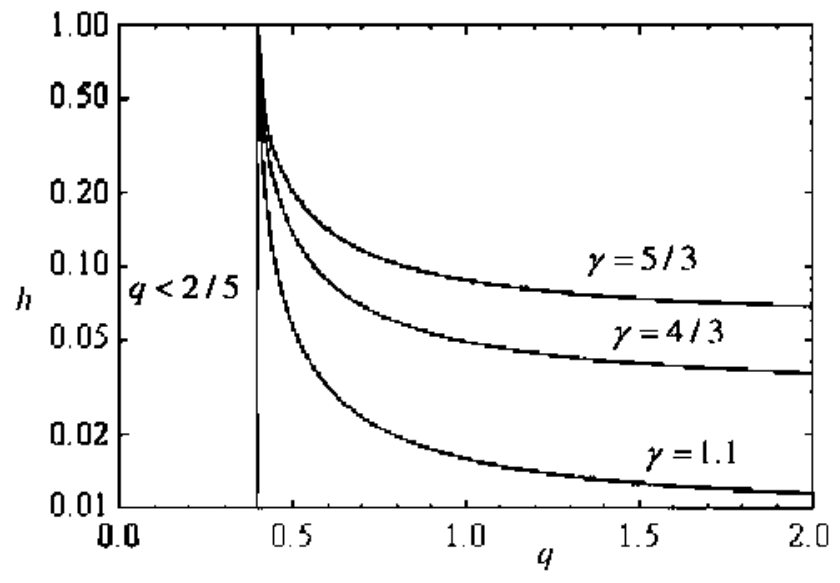

Fig. 5 Normalized thickness of the shell as a function of $q$

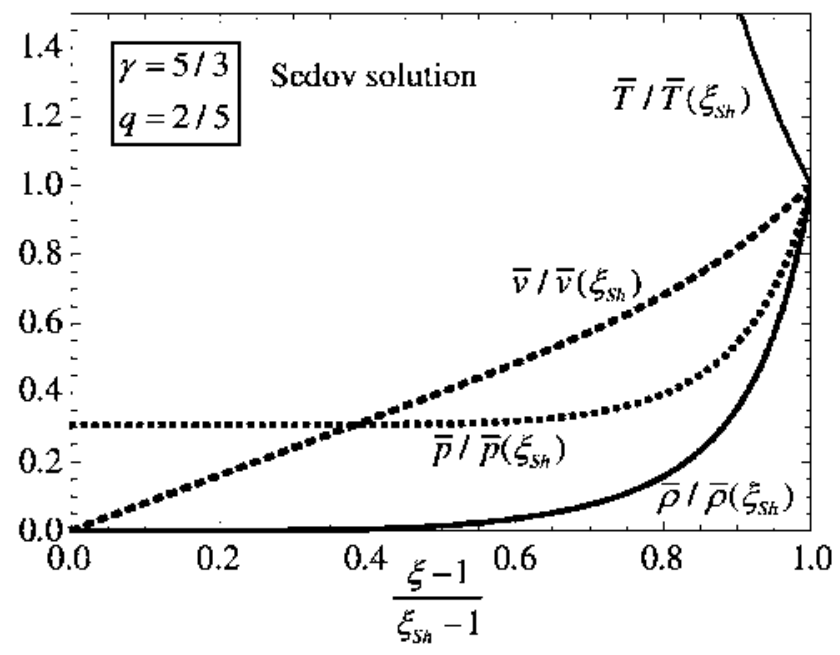

Fig. 6 Variables in the Taylor-Sedov low $\int 4 \pi\left(R_{P_{i s t}}(t)\right)^{2} P_{l n t}(t) d R_{p_{i s t}}=E_{0}\left(t / t_{0}\right)^{5 q-2}$ where $E_{0}$ and $t_{0}$ are two constants. The time-dependence in $E(t)$ cancels for $q=2 / 5$ (Sedov-Taylor instantaneous point explosion) and the encrgy $E_{0}$ is a constant of motion in addition to the expression $F_{0}=\left(1 /\left[\bar{\rho}(\xi) \xi^{2}[\bar{v}(\xi)-\xi]\right]\right)(\bar{T}(\xi) /$ $\left.[\bar{\rho}(\xi)]^{\gamma-1}\right)^{3 q /[2(q-1)]}$ that can be derived from (2)-(4) with no constraint for $\gamma$ and $q$, and that combines both the entropy and the mass flux.

Finally, the mass of the shell, $M(t)$, increases like $t^{3 /}$ and its non-dimensionless thickness, $H(t)=h R p_{i s t}(t)$, varies as $t^{q}$. For $q>1$, the accretion rate is high enough to produce a mass accumulation at the piston surface with a diverging density (see above). Moreover, as $\bar{\rho}(1) \propto(\xi-1)^{-\mu}$, the more compressibility effects are important (i.e. $\gamma \rightarrow 1$ ), the sharper the density peak is ( $\mu \rightarrow \mu_{\max }$ ).

\section{Stability analysis}

In this section, assuming arbitrary $q$ and an adiabatic shocked region (Vishniac 1983), we perform the 3D linear stability analysis of the time-dependent 1D background solution. First of all, the physical quantities are written as $Q(r, t)+\delta Q(r, \theta, \phi, t)$ where $Q(r, t)$ stands for any of the background variables (velocity, density...) and where $\delta Q(r, \theta, \phi, t)$ corresponds to the associated perturbation. Second, the $\delta Q$ 's are expanded in spherical harmonics $Y_{l, m}(\theta, \phi)$ and we linearize the mass, momentum (radial and transversal) and entropy conservation equations. The perturbations cannot behave exponentially with time as the unperturbed background solution is not steady. However, as noticed by Ryu and Vishniac (1987), the lime variable, $t$, can be separated writing perturbations in the form $\delta Q(r, \theta, \phi, t) \propto t^{\sigma} \bar{Q}_{1}(\xi) Y_{l, m}(\theta, \phi)$ where $\bar{Q}_{1}(\xi)$ is the first order perturbation of the self-similar quantity $\bar{Q}(\xi)$. The exponent $\sigma$ represents a dimensionless "complex growth rate" and the perturbed system is unstable if $\sigma_{R} \equiv \operatorname{Re}(\sigma)>0$. The lincarized system contain six periurbed variables $\bar{Q}_{1}$, i.c. the four fluid variables, density $\bar{\rho}_{1}(\xi)$, temperature $\bar{T}_{1}(\xi)$, radial velocity $\bar{v}_{1}(\xi)$, and the transverse divergence of the transverse velocity $\bar{w}_{1}(\xi)$, and morcover the perturbed positions of piston, $\bar{\xi}_{\text {Pist }, 1}$, and shock interface $\bar{\xi}_{S h, 1}$. We arrive then to a linear homogeneous $4^{\text {th }}$ order system of ODE's for the fluid variables. The solution of this system (with 4 arbitrary constant of integration, $\left.C_{j} ; j=1, \ldots, 4\right)$ must fulfill the following six linear homogeneous boundary conditions involving six unknown $\left(C_{j}, \bar{\xi}_{P_{i s t .1}}, \bar{\xi}_{S h, 1}\right)$ : the 4 Rankine-Hugoniot relations at the shock interface $\left(\xi=\xi_{S h}\right)$, and at the piston interface $(\xi=1)$, pressure must be conlinuous and radial velocity equals to radial piston velocity. The compatibility condition for this linear-homogeneous algebraic system provides the dispersion relation for $\sigma, D(\sigma, l, \gamma, q)=0$, where 
$l$ is the Legendre index mode. Notice that $\sigma$ does not depends on the azimuthal number $m$ as the background zero order solution has radial symmetry.

For $q>1$, the accelcrated shell (sec Fig. 3 ) experiences a positive and infinite density jump at the piston interface $r=$ $R_{P i s t}(t)$ (notice that the density is zero for $r<R_{P i s t}(t)$ ) and hence it is expected to be Rayleigh-Taylor unstable (Lord Raylcigh 1883; Taylor 1950; Bernstein and Book 1978; Sanz 1994; Ribeyre et al. 2007). This result is exhibited in Fig. 7 where the real (unstable) branch, $\sigma_{R}$, of $\sigma$ is plotted as a function of the mode number $l$. It turns out that low modes $\left(l \leq l_{c} \approx 1 /(q-1)\right)$ do not give rise to RTI but above the threshold $l_{c}, \sigma_{R}(l)$ is monotonically increasing with $l$. On the other hand, notice the very weak influence of the compressibility ( $\gamma$-effects) on the growth rate. As expected, at large number $l, \sigma_{R}$ is independent of $\gamma$. In addition, an asymptotic expression of $\sigma_{R}$ can be estimated in terms of $l$. Indeed, from the linear differential equation governing the rippled amplitude of the piston position, $\delta R_{P_{i s t} t}(\theta, \phi, t)$, which is $\partial^{2}\left(\delta R_{P_{i s t}}\right) / \partial t^{2} \approx k R_{\text {Pist }}^{\prime \prime}(t) \delta R_{P_{i s t}} \approx$ $\left[\sqrt{l(l+1)} R_{\text {Pist }}^{\prime \prime}(t) / R_{\text {Pist }}(t)\right] \delta R_{\text {Pist }}$ where $k \propto l / R_{\text {Pist }}(t)$ is the wave number of the perturbation, it can be derived analytically that $\sigma_{R}(l) \propto\lfloor q(q-1) l]^{1 / 2}$ where the exposant $1 / 2$ is a specific property of RTI. This asymptotic expression has been also plotted in Fig. 7 to show the correct behavior of the numerical results.

The decelerated case $(q<1)$ appears to be more complex but more instructive. First of all, as the shock is decelerated and as at its location the mass density $\rho$ drops from $\rho_{S h}=\lfloor(\gamma+1) /(\gamma-1)] \rho_{0}$ to $\rho_{0}$, we could expect that the moving surface of the sphere $r=R_{S h}(t)$ is submitted to RTI. This point is however not quite clear up to now since this surface experiences a mass flow and, as a consequence, does not behave as a real interface between two media. This behavior will be examined in further studies although it is taken into account in the dispersion relation we are going to show in

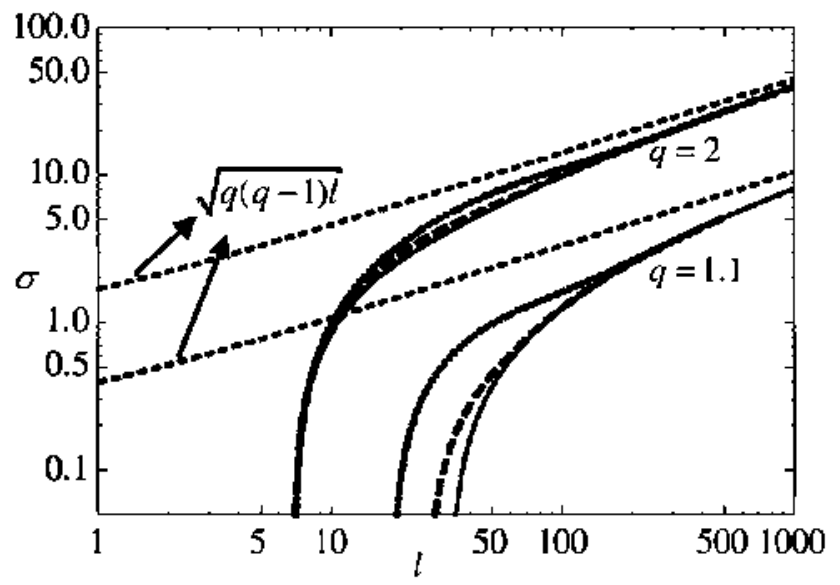

Fig. 7 Dispersion relation in the accelerated case for $q=1.1$ and $q=2$ for three values of $\gamma(-\gamma=5 / 3, \cdots \gamma=4 / 3$ and $-\gamma=1.1)$ the following. Second, within the deceleraled shell the density increases monotonically with the radius and it could be argued that internal RT unstable modes could not be excited. This conclusion is right for a constant deceleration but, as in our case, it decreases with time (we remind that both faces of the shell decelcrate like $t^{q-2}$ ), RTI may occur. This behavior is similar to that of a vertical pendulum experiencing gravity with a time-decreasing magnitude. In that case, the amplitude of the oscillation increases with time and the pendulum performs unstable oscillations. As a result, although one of the possible physical mechanism driving this instability was pointed out by Vishniac and Ryu (Vishniac 1983; Ryu and Vishniac 1987, 1988; Vishniac and Ryu 1989) as the ram pressure, $\rho_{0}\left[R_{S h}^{\prime}(t)\right]^{2}$, on the rippled shock front, this is not the only process.

These results are shown in Figs. 8 and 9 where we have plotted $\sigma_{R}(l)$ for $q=1 / 2$ and $q=2 / 5$ (Sedov-Taylor solution), respectively, for several values of $\gamma$. In opposition to the accelerated case, the growth rate is very sensitive to this parameter and provided $\gamma$ satisfies $\gamma>1, \sigma_{R}$ reaches a maximum value, $\sigma_{R, \max }$, for a finite value of $l$ that depends upon $\gamma$. However, $\sigma_{R, \max }$ is much weaker than the RTI growth rate of the accelerated shell (sec Fig. 7), except for extremely small and unrealistic values of $\gamma$. Actually, $\sigma_{R, \max }$ is only larger than unity for $\gamma$ smaller than 1.05 roughly and the instability vanishes $\left(\sigma_{R}<0\right)$ if $\gamma>1.1$ (resp. $\gamma>1$.2) for $q=1 / 2$ (resp. $q=2 / 5$ ).

In the same figures, we have plotted too the analytical expression [(*)-curves] of the growth rate obtained by Vishniac and Ryu in the infinitely thin-shell approximation (Vishniac 1983; Ryu and Vishniac 1987; Vishniac and Ryu 1989). Each upper (*)-curve in Figs. 8 and 9 corresponds to the situation studied by Vishniac and Ryu (Vishniac 1983; Ryu and Vishniac 1987) and it is clear that in both figures these curves are obtained from our study in the asymptotic limil $\gamma \rightarrow 1$. This result is not surprising since for an almost isothermal shock the ratio $\rho_{S h} / \rho_{0}$ goes to infinity and any

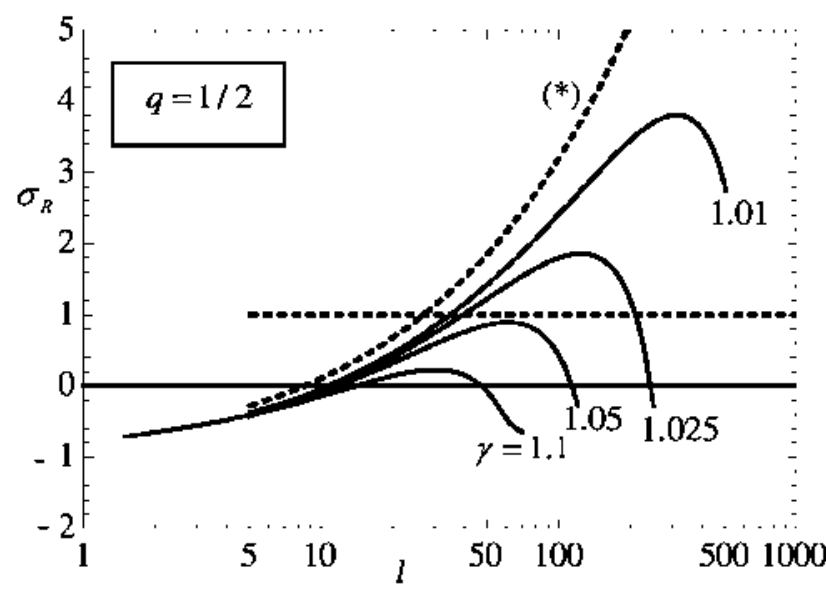

Fig. 8 Dispersion relation for $q=1 / 2$ for various values of $\gamma$ 


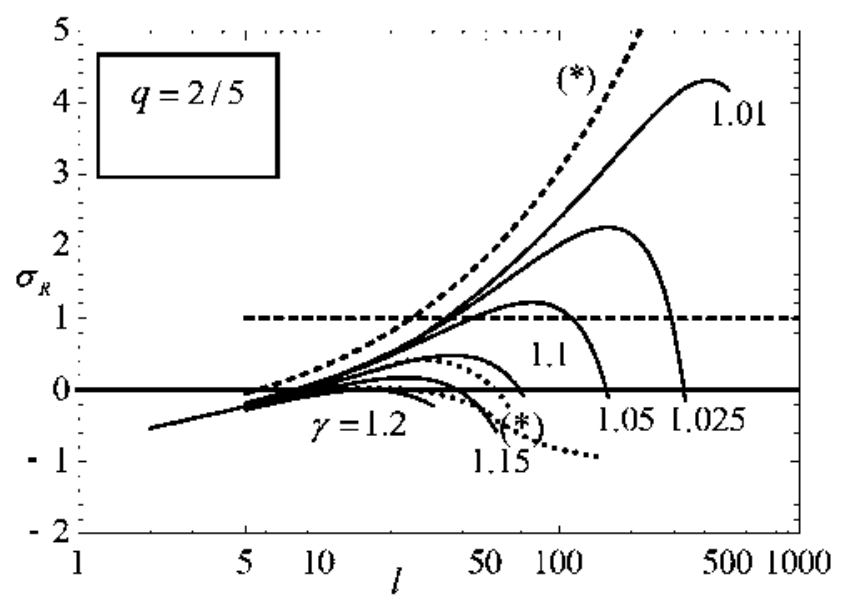

Fig. 9 Dispersion relation for $q=2 / 5$

finite mass of accreted material will be contained within a shell of infinitely small thickness.

In addition, Fig. 9 exhibits two more $\left({ }^{*}\right)$-curves (dotted lines) obtained by Vishniac and Ryu (1989) for $\gamma=1.1$ and $\gamma=1.2$ in a isothermal approximation. Compared to our results, the growth rate derived by Vishniac and Ryu (1989) is lower (resp. higher) than the one we have computed for $\gamma=1.1$ (resp. $\gamma=1.2$ ). This behavior is similar to that obtained by these authors in their Figs. 1a and 1b where they make a comparison between the results of their isothermal approximation (1989) with the results of an exact solution.

\section{Conclusion}

In this paper we have studied the evolution and the dynamic stability of a shell expanding like $t^{q}$ in a uniform density medium. The shell in bounded by a strong shock at its outer face and it experiences a uniform time-dependent pressure on its inner border. Taking into account these well-defined boundary conditions, the dispersion relation has been obtained for both decelerated and acceleraled shells. Although in the two cases RTI oceur, decelerated shells appear to be much more stable with a cul-ofl for the mode number. However for $\gamma \rightarrow 1$, the growth rate of the instability increases monotonically with $l$, describing therefore the thin shell approximation studied earlier by Vishniac and Ryu (Vishniac 1983; Ryu and Vishniac 1987). A comparison with the results derived by these authors in the isothermal approximation (Vishniac and Ryu 1989) has been also performed. More generally, our work is an extension of these former studies for arbitrary exponent $q$ and polytropic index $\gamma$.

\section{References}

Bernstein, I.B., Book, D.L.: Astrophys. J. 225, 633 (1978)

Cavel, C., Nguyen, H.C., Michaul, C., Falize, E., Bouquet, S., di Menza, L.: Astrophys. Space Sci. 322, 91 (2009)

Cavet, C., Michaut, C., Roy, F., Nguyen, H.C., Bouquet, S., Sauty, C.: Astrophys. Space Sci. (2010) this issue

Chevalier, R.A.: Astron. Astrophys. 15, 175 (1977)

Chevalier, R.A.: Astrophys. J. 258, 790 (1982)

Chevalier, R.A., Blondin, J.M.: Astrophys. J. 444, 312 (1995)

Chevalier, R.A., Blondin, J.M., Emmering, R.T.: Astrophys. J. 392 , 118 (1992)

Gull, S.F.: Mon. Not. R. Astron. Soc. 161, 47 (1973)

Klcin, R.I., Woods, D.T.: Astrophys. J, 499, 777 (1998)

Mac Low, M.-M., Norman, M.L.: Astrophys. J. 407, 207 (1993)

Nadyozhin, D.K.: Astrophys. Space Sci. 112, 225 (1985)

Rayleigh, Lord: Proc. Lond. Math. Soc. 14, 170 (1883)

Ribcyre, X., Hallo, L., Tikhonchuk, V.T., Bouquet, S., Sanz., J.: Phys. Plasmas 14, 112902 (2007)

Ryu, D., Vishniac, E.T.: Astrophys. J. 313, 820 (1987)

Ryu, D., Vishniac, E.T.: Astrophys. J. 331, 350 (1988)

Ryu, D., Vishniac, E.T.: Astrophys. J. 368, 411 (1991)

Sanz, J.: Phys. Rev. Lett. 73, 2700 (1994)

Sedov, L.: Similarity and Dimensional Methods in Mechanics. Academic Press, New York (1959)

Taylor, G.: Proc. R. Soc. Lond. 201, 192 (1950)

Velazquez, P.F., Gomez, D.O., Dubner, G.M., Gimenez de Castro, G., Costa, A.: Astron. Astrophys. 334, 1060 (1998)

Vishniac, E.T.: Astrophys. J. 274, 152 (1983)

Vishniac, E.T.: Astrophys. J. 428, 186 (1994)

Vishniac, E.T., Ryu, D.: Astrophys. J. 337, 917 (1989)

Wang, C.-Y., Chevalier, R.A.: Astrophys. J. 549, 1119 (2001)

Woltjer, L.: Astron. Astrophys. 10, 129 (1972) 